\title{
Publisher Correction: Twinfilin uncaps filament barbed ends to promote turnover of lamellipodial actin networks
}

Markku Hakala (D), Hugo Wioland (D), Mari Tolonen, Tommi Kotila (D), Antoine Jegou (D), Guillaume Romet-Lemonne (D) and Pekka Lappalainen (D)

Correction to: Nature Cell Biology https://doi.org/10.1038/s41556-020-00629-y, published online 8 February 2021

In the version of this Article originally published, there were several typographical errors. In Fig. 2b,d, 'flow' was cut off in 'retrograde flow'. Instances of 'normalized fluorescence' on the $y$ axes of Figs. $4 \mathrm{i}, \mathrm{h}$ and $8 \mathrm{c}$, d were missing letters. In Fig. $6 \mathrm{c}$, the confidence interval was not centered on the blue dashed line in sample ' $2.7 \mu \mathrm{M}$ TWF1 $+37 \mu \mathrm{M}$ V1', and ' $\times 7$ ' should be ' $\times 6$ ' (shown below). In Fig. $7 \mathrm{~d}$, 'mCherry-TWF1 domain' was written as 'mCherry-TWF1 doma+in'. 'Twinfilin' was missing letters in Fig. 8e,g, and 'cofilin' was cut off in Fig. 8e as well. In the sentence "These pauses are due, at least in part, to multiple surface-anchoring points," the commas were incorrectly formatted as single quotes. In Extended Data Fig. 3a, the lower row of cell images was missing the information about what is shown in the panels. This should be, from left to right: Phalloidin/Transferrin/DAPI, Phalloidin, Transferrin. Finally, In Extended Data Fig. 3b, information for what is shown on the $x$ and $y$ axes was missing: $x$ axis from left to right: B16-F1 wt, Twf1/Twf2-KO-g3. $y$ axis: correlation coefficient. The errors have been corrected. 
a

(1) Polymerization (10 min)

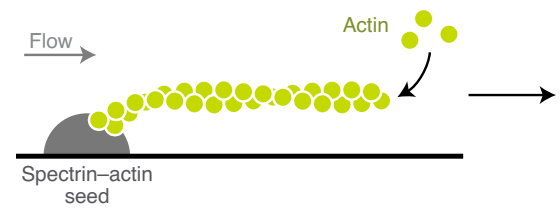

b

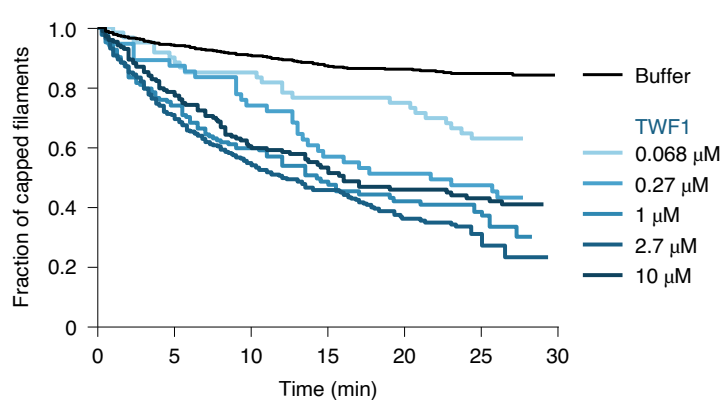

Original

(2) Capping (2 min)
(3) Observation (30 min)

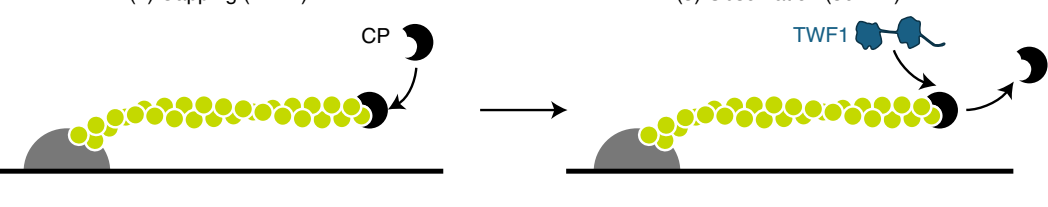

c

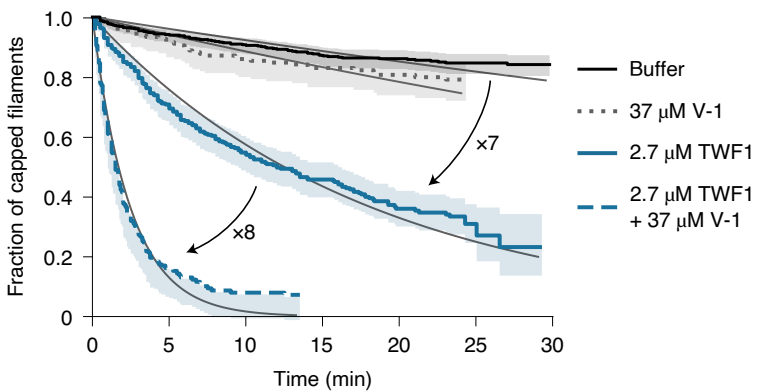

Corrected

a

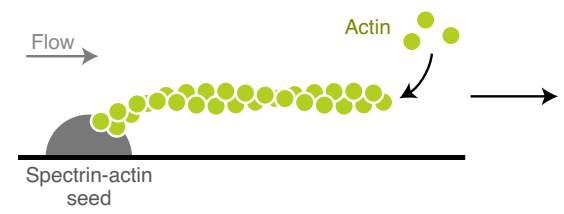

b

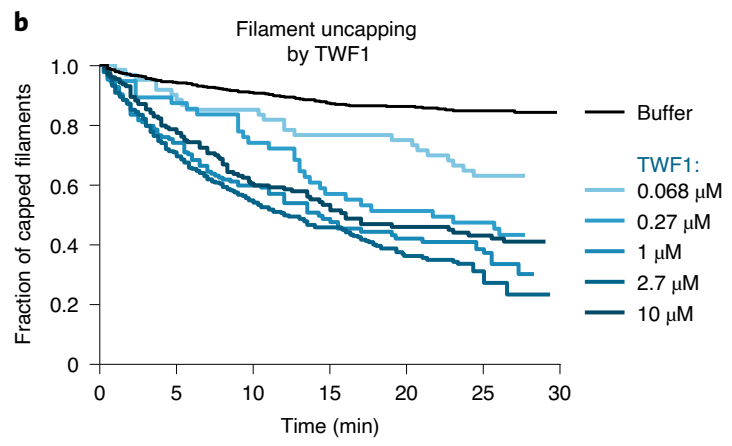

2. Capping (2 min)

Capping protein
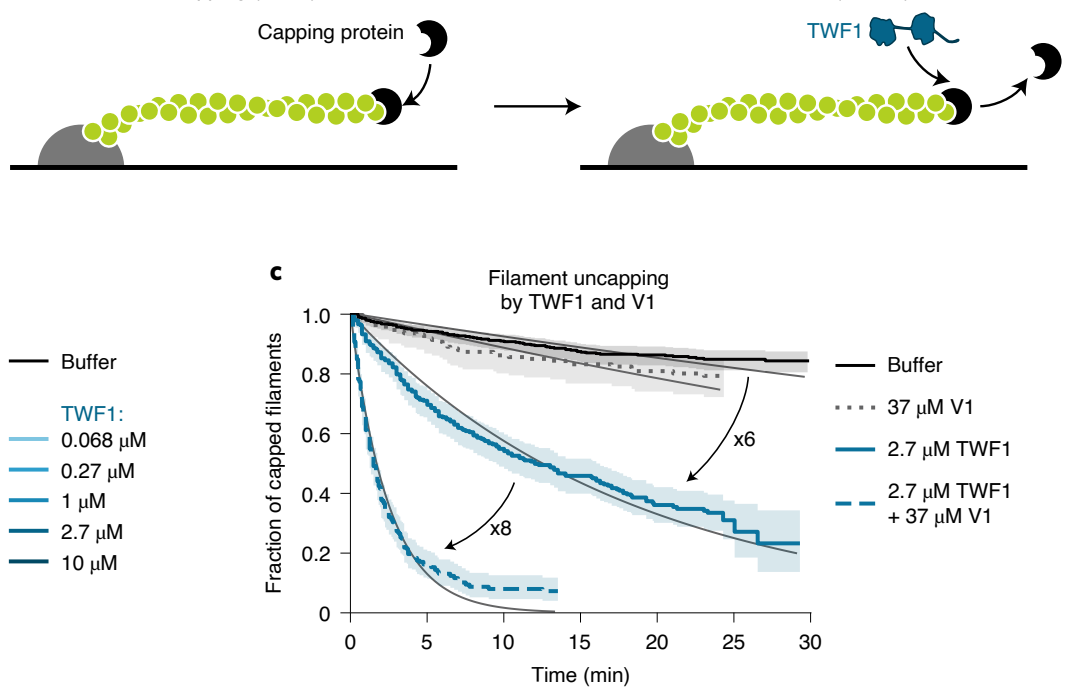

- Buffer

... $37 \mu \mathrm{M}$ V1

- $2.7 \mu \mathrm{M}$ TWF1

$-2.7 \mu \mathrm{M}$ TWF1

$+37 \mu \mathrm{M}$ V1

Fig. 6 | Original and Corrected.

Published online: 2 March 2021

https://doi.org/10.1038/s41556-021-00651-8

(c) The Author(s), under exclusive licence to Springer Nature Limited 2021 